\title{
Processamento Auditivo Central em Pessoas Idosas: Resultados Preliminares
}

\section{Central Auditory Processing in Elderly People: Preliminary Results}

Carolina Fernandes ${ }^{1,2}$, Carla Matos Silva ${ }^{2,3}$, Ana Rita Alves ${ }^{2,4}$, Cláudia Prata ${ }^{2,5}$, Clara Rocha6,7, Jorge Humberto Martins (On behalf of the AGA@4life Consortium)

Autor Correspondente/Corresponding Author: Carolina Fernandes [carol_sofia_fernandes@hotmail.com] Av. Padre Américo, n. ${ }^{\circ}$ 580, 3220-179 Miranda do Corvo, Coimbra, Portugal ORCID iD: 0000-0002-5037-6749

\section{RESUMO}

INTRODUÇÃO: A presbiacúsia acarreta dificuldades na interpretação do sinal sonoro com uma influência acrescida na perceção do discurso na presença de ruído. Pretende-se estudar as habilidades do processamento auditivo central na discriminação da fala no ruído, em pessoas idosas.

MATERIAL E MÉTODOS: A amostra foi constituída por 20 sujeitos, com idades compreendidas entre os 67 e os 92 anos. Todos realizaram otoscopia, timpanograma, audiograma tonal simples e teste de fala no ruído. Este estudo é do tipo descritivo-correlacional e transversal.

RESULTADOS: A curva audiométrica apresenta declínio significativo ( $p<0,001)$ com o aumento das frequências testadas, a partir da frequência dos $500 \mathrm{~Hz}$, apresentando o ouvido esquerdo melhores resultados do que o ouvido direito, com significância estatística ( $p=0,043)$, apenas nos $250 \mathrm{~Hz}$. No teste de fala no ruído, os resultados do ouvido esquerdo evidenciaram uma média de acertos de $21,5 \%$ na relação sinal/ruído de $10 \mathrm{~dB}$, tendo um aumento significativo $(p<0,001)$ na relação sinal/ruído de 15 dB cuja média de acertos foi 26,3\%. O ouvido direito exibe uma média de acertos de 17,3\% e de 27,85\% na relação sinal/ruído de 10 dB e de 15 dB respetivamente, com um aumento significativo ( $p=0,002)$ na relação sinal/ruído de $15 \mathrm{~dB}$.

CONCLUSÃO: Tendo em conta os resultados, em que o teste de fala no ruído apresenta uma média de acertos inferior aos 50\% em ambos os ouvidos, chegámos à conclusão que quanto menor for a relação sinal/ruído maiores as dificuldades na compreensão da fala. Assim, a avaliação audiológica da pessoa idosa deve incluir a avaliação periférica e central.

1. Audiologista na Minisom uma marca amplifon, Lisboa, Portugal, BSc. 2. Instituto Politécnico de Coimbra, ESTeSC - Coimbra Health School, Departamento de Audiologia, Coimbra, Portugal, PhD. 3. Faculdade de Letras, Centro de Linguística da Universidade de Lisboa, Universidade de Lisboa. 4. Audiologista no Centro Cirúrgico de Coimbra, Coimbra, Portugal, BSc. 5. Audiologista no O Meu Doutor, Reabilitação Auditiva Portugal, Portugal, BSc. 6. Instituto Politécnico de Coimbra, ESTeSC Coimbra Health School, Departamento das Ciências Complementares, Coimbra, Portugal, PhD. 7. INESC Coimbra, Portugal. 8. Audiologista no Serviço ORL do Centro Hospitalar e Universitário de Coimbra - Hospital Geral e Hospital Pediátrico, PhD.

• Autor (es) (ou seu (s) empregador (es)) 2019. Reutilização permitida de acordo com CC BY-NC. Nenhuma reutilização comercial. ${ }^{\bullet}$ Author(s) (ortheir employer(s)) 2019. Re-use permitted under CCBY-NC. No commercial re-use. 
PALAVRAS-CHAVE: Doenças Auditivas Centrais; Idoso; Perda Auditiva Neurossensorial; Perturbações da Perceção Auditiva

\section{ABSTRACT}

INTRODUCTION: Presbycusis entails difficulties in the interpretation of the sound signal with an increased influence in perceiving speech in the presence of noise. We intend to study the central auditory processing skills in speech discrimination in noise of elderly people.

METHODS: The sample consists of 20 people, aged from 67 to 92. All the people have undergone an otoscopy, a tympanogram, a pure tone audiogram and the speech in noise test. This is a descriptive-correlational study.

RESULTS: The audiometric curve shows a statistically significant decline ( $p<0.001)$ with increasing in the frequencies tested, from the frequency of $500 \mathrm{~Hz}$. The left ear presents better results than the right ear, with statistical significance ( $p=0.043)$, only at the frequency of $250 \mathrm{~Hz}$. In the speech in noise test, the results from the left ear showed an average of $21.5 \%$ correct answers with a signal/noise relation of $10 \mathrm{~dB}$, with a statistically significant increase $(p<0.001)$ with signal/noise relation of $15 \mathrm{~dB}$, where the average of correct answers was $26,3 \%$. For the right ear, we obtained an average of $17.3 \%$ and $27.85 \%$ of correct answers with a signal/noise relation of $10 \mathrm{~dB}$ and $15 \mathrm{~dB}$, respectively. There was also a significant increase $(p=0.002)$ with a signal/noise relation of $15 \mathrm{~dB}$.

CONCLUSION: Considering that the speech in noise test exhibits a mean of less than 50\% for both ears, we concluded that the smaller the signal/noise relation the greater the difficulties in understanding the speech. So the audiological evaluation of the elderly should include the peripheral and central evaluation.

KEYWORDS: Aged; Auditory Diseases, Central; Auditory Perceptual Disorders; Hearing Loss, Sensorineural

\section{INTRODUÇÃO}

O envelhecimento da população é cada vez mais, uma realidade não só a nível nacional mas também, a nível mundial. ${ }^{1}$ Este é caracterizado por um conjunto de alterações fisiológicas, biológicas e psicológicas que se encontram associadas ao avançar dos anos. ${ }^{2}$ A perda de audição associada à idade, presbiacúsia, resulta da degradação bilateral e progressiva das células ciliadas do ouvido interno, que afeta principalmente a zona basal da cóclea e que por sua vez, irá influenciar a perceção auditiva da pessoa, com repercussões na sua qualidade de vida. ${ }^{1-3}$ Prevê-se que esta diminuição da acuidade auditiva afete mais de 60\% dos indivíduos com idade igual ou superior a 60 anos, fazendo deste o terceiro problema com maior incidência na população geriátrica. ${ }^{2-4}$

O sistema auditivo é uma peça fundamental no desenvolvimento da comunicação oral, pelo que, qualquer perturbação que possa existir, quer a nível periférico quer a nível central, leva ao comprometimento na capacidade de manter um discurso coerente, causando uma dificuldade acrescida na aquisição e/ou do processamento do estímulo sonoro. ${ }^{3} \mathrm{O}$ processamento auditivo central (PAC) é responsável pelo processamento da informação auditiva no sistema nervoso central e pela atividade neurobiológica implicada. 5,6 Engloba maioritariamente as estruturas desde a via auditiva até ao córtex, que são responsáveis pela realização de várias habilidades das quais o indivíduo depende para interpretar os sons verbais e não-verbais que ouve.,

A existência de dificuldades na perceção do discurso na população mais velha, é bastante comum principalmente quando há ruído ambiente ${ }^{1,7,8}$ devido à influência do envelhecimento, quer ao nível do sistema auditivo periférico quer ao nível do processamento auditivo central, ${ }^{9}$ como consequência das alterações nas áreas centrais do sistema nervoso auditivo. ${ }^{4,5}$ Considerando que as dificuldades de perceção da fala é um dos aspetos mais referido pela população pessoa idosa torna-se pertinente a avaliação do processamento auditivo central utilizando para o efeito uma adequada seleção dos testes que depende de vários fatores, designadamente dos objetivos do profissional, tendo por base uma anamnese rigorosa que pode orientar o Audiologista para a(s) habilidade(s) comprometida(s). ${ }^{10,11}$

Em Portugal, a avaliação do processamento auditivo central tem sido pouco valorizada. Atualmente a avaliação da audição da pessoa idosa restringe-se à avaliação do sistema auditivo periférico com vista à implementação de programas de reabilitação auditiva, descorando a avaliação do processamento auditivo central que, uma vez incluída poderia otimizar os benefícios desses mesmos programas e, poderia ajudar a perceber o modo como o envelhecimento pode influenciar as habilidades auditivas, ${ }^{10-13}$ encaminhando, quando necessário, estes 
indivíduos para um plano de treino auditivo que visa a estimulação da via auditiva, com o objetivo de maximizar a neuroplasticidade do sistema nervoso central. ${ }^{14-18}$ Neste sentido surgiu o presente estudo que tem como objetivo estudar as habilidades do processamento auditivo central na discriminação da fala no ruído, num grupo de pessoas idosas que frequentam um centro de dia da região centro do país. Na génese deste estudo estão algumas questões de investigação, nomeadamente: Qual o desempenho destes idosos no teste de fala no ruído? Os idosos com maior perda tonal média apresentam piores resultados no teste de fala no ruído?

\section{MATERIAL E MÉTODOS}

Neste estudo participaram 38 pessoas idosas que frequentam um centro de dia da região centro do país. Após o cumprimento dos requisitos éticos, nomeadamente a obtenção por escrito do termo de consentimento informado livre e esclarecido, foi feita uma avaliação audiológica periférica dos indivíduos através da otoscopia, do timpanograma, do audiograma tonal simples nas frequências de $250 \mathrm{~Hz}, 500 \mathrm{~Hz}, 1000 \mathrm{~Hz}, 2000 \mathrm{~Hz}$, $4000 \mathrm{~Hz}$ e $8000 \mathrm{~Hz}$ e ainda, com o intuito de avaliar a parte central do sistema auditivo no que diz respeito à discriminação da fala no ruído, foi realizado o teste de fala no ruído com uma relação sinal/ruído de 10 dB e de $15 \mathrm{~dB} \cdot{ }^{19,20}$

No teste de fala no ruído foram usados dissílabos de uma lista foneticamente equilibrada, com a fala em apresentação ipsilateral e com ruído competitivo, com uma relação sinal/ruído de $+10 \mathrm{~dB}$ e $+15 \mathrm{~dB}$. A fala (sinal) é apresentada sempre à mesma intensidade - $50 \mathrm{~dB}$ acima do valor da perda tonal média, aferido anteriormente com um audiograma tonal simples, enquanto o ruído é apresentado $10 \mathrm{~dB}$ ou $15 \mathrm{~dB}$ abaixo do sinal. Podem ser usados diferentes tipos de ruído, sendo o mais usado o ruído branco. Neste estudo, o tipo de ruído usado foi uma gravação de conversas tentando simular o ambiente real a que este tipo de sujeitos está submetido diariamente. Na realização do teste é solicitado ao indivíduo para repetir cada uma das palavras ouvidas.

O critério de referência de normalidade para a habilidade de fecho auditivo neste teste é obter acertos superiores a $70 \%$ para ambos os ouvidos. ${ }^{21}$

Foram considerados como critérios de exclusão: idade inferior a 65 anos, a presença de cerúmen obliterante, de timpanograma do tipo B e a perda tonal média superior a $50 \mathrm{dBHL}$. Este último critério deve-se ao facto de não ser possível realizar o teste de fala no ruído, independentemente da relação sinal/ruído, dado que o estí- mulo sonoro é apresentado a $50 \mathrm{dBHL}$ acima da perda tonal média. Neste estudo foram excluídos dois indivíduos por terem idade inferior a 65 anos, quatro indivíduos com cerúmen obliterante, três indivíduos com timpanograma do tipo B e nove que apresentavam perda auditiva de grau severo a profundo. Todos os indivíduos com cerúmen obliterante e com timpanograma do tipo B foram encaminhados para consulta de Otorrinolaringologia. A amostra foi recolhida entre o dia 20 e o dia 23 de março de 2018.

Este estudo é do tipo descritivo-correlacional e transversal. Foi considerada como variável dependente os resultados obtidos no audiograma tonal simples e no teste de fala no ruído. A variável independente são os idosos que frequentam este centro de dia da região centro do país.

A análise estatística foi realizada com recurso ao software IBM $^{\circledR}$ SPSS $^{\circledR}$ v. 24.0. Na análise descritiva foram determinados a média e o desvio padrão (DP) para as variáveis quantitativas, e frequências para as variáveis qualitativas. A avaliação da normalidade de distribuição das variáveis quantitativas foi feita de acordo com o teste Shapiro-Wilk. As comparações entre os dois ouvidos (direito e esquerdo) e entre as relações sinal/ruído 10 dB e 15 dB foram feitas segundo o teste $t$ - Student para amostras emparelhadas (no caso de se verificar a normalidade da distribuição subjacente) ou, segundo o teste Wilcoxon (no caso contrário).

As comparações entre as frequências, $250 \mathrm{~Hz}, 500 \mathrm{~Hz}$, $1000 \mathrm{~Hz}, 2000 \mathrm{~Hz}, 4000 \mathrm{~Hz}$ e $8000 \mathrm{~Hz}$ foram feitas segundo o teste não paramétrico Friedman e as comparações múltiplas foram feitas de acordo com o teste não paramétrico Wilcoxon.

Considerou-se uma significância de 0,05 para todas as comparações.

\section{RESULTADOS}

Num total de 38 pessoas com idade igual ou superior a 65 anos de idade, apenas 20 pessoas idosas, com idades compreendidas entre os 67 e os 92 anos e média de idades de 82,9 anos (DP = 7,9), 17 são mulheres (70\%), fizeram parte da amostra devido aos critérios de exclusão pré-estabelecidos e mencionados anteriormente. Verifica-se que os seus limiares auditivos variam entre os 23,5 dB (DP = 11,6 dB), obtido aos $250 \mathrm{~Hz}$ no ouvido esquerdo, e os 71,5 dB (DP = 21,2 dB), obtido aos $8000 \mathrm{~Hz}$ no ouvido direito (Fig. 1).

A curva audiométrica evidencia efeitos significativos na frequência, com declínio a partir da frequência de 


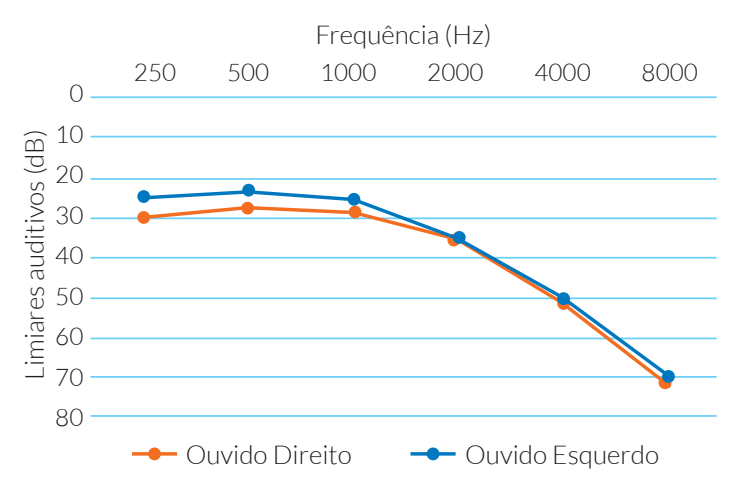

FIGURA 1. Média dos limiares auditivos para ambos os ouvidos nas frequências testadas. ${ }^{*} p<0,05$.

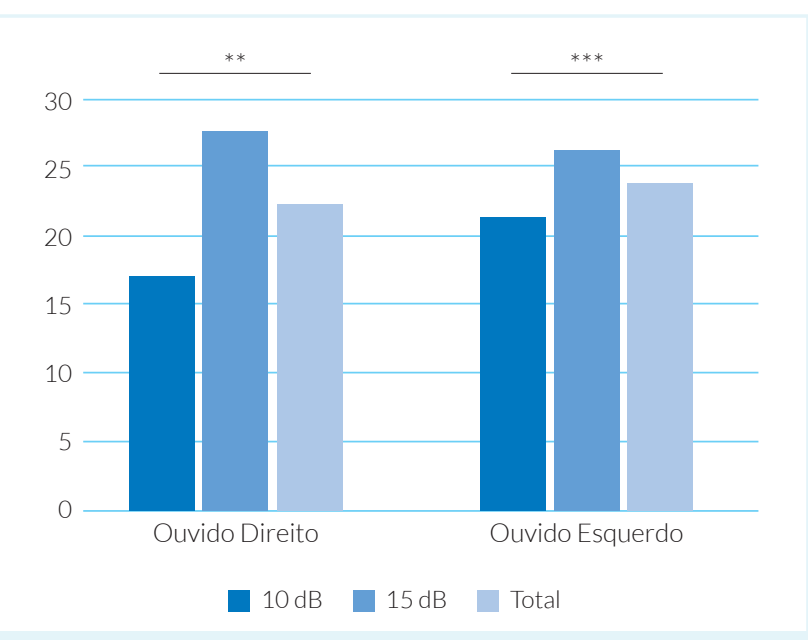

FIGURA 2. Percentagem média de acertos segundo a relação sinal/ruído para ambos os ouvidos no Teste de fala no ruído. ${ }^{* *} p<0,01 ;{ }^{* * *} p<0,001$

TABELA 1. Correlação entre o teste de fala no ruído segundo a relação sinal/ruído de $10 \mathrm{~dB}, 15 \mathrm{~dB}$ e total e a perda tonal média (PTM) para ambos os ouvidos.

\begin{tabular}{|l|c|c|c|c|}
\hline & \multicolumn{2}{|c|}{ OD } & \multicolumn{2}{c|}{ OE } \\
\hline & $\rho$ & $p$ & $\rho$ & $p$ \\
\hline $10 \mathrm{~dB}$ & $-0,676$ & $<0,001$ & $-0,733$ & $<0,001$ \\
\hline $15 \mathrm{~dB}$ & $-0,683$ & $<0,001$ & $-0,733$ & $<0,001$ \\
\hline Total & $-0,706$ & $<0,001$ & $-0,726$ & $<0,001$ \\
\hline
\end{tabular}

$500 \mathrm{~Hz}$ ( $p<0,001$ para ambos os ouvidos). No ouvido esquerdo verificou-se um aumento significativo dos limiares auditivos dos $250 \mathrm{~Hz}$ para os $2000 \mathrm{~Hz}(p=0,009)$ sendo que, em ambos os ouvidos se verificou um aumento significativo dos limiares auditivos ( $p<0,001)$, tanto dos $250 \mathrm{~Hz}$ para os $4000 \mathrm{~Hz}$ como para os $8000 \mathrm{~Hz}$. Este aumento significativo dos limiares foi de igual modo observado, uma vez mais em ambos os ouvidos, dos $500 \mathrm{~Hz}$ para os $2000 \mathrm{~Hz}$ ( $p=0,017$ e $p=0,002$, respetivamente), para os $4000 \mathrm{~Hz}$ ( $p<0,001$ ) e para os $8000 \mathrm{~Hz}$ $(p<0,001)$; dos $1000 \mathrm{~Hz}$ para os $2000 \mathrm{~Hz}(p=0,021 \mathrm{e}$ $p=0,002$ respetivamente), para os $4000 \mathrm{~Hz}(p<0,001)$ e para os $8000 \mathrm{~Hz}(p<0,001)$; dos $2000 \mathrm{~Hz}$ para os
$4000 \mathrm{~Hz}(p<0,001)$ e para os $8000 \mathrm{~Hz}(p<0,001)$; bem como dos $4000 \mathrm{~Hz}$ para os $8000 \mathrm{~Hz}(p<0,001)$, sustentando estatisticamente a informação qualitativa do padrão de variação dos limiares auditivos em função da frequência representados na Fig. 1.

Os efeitos localizam-se predominantemente entre as frequências mais extremas do sinal, ou seja, entre os 250 e os $500 \mathrm{~Hz}$ e os 4000 e $8000 \mathrm{~Hz}$, em ambos os ouvidos. De referir ainda a existência de diferenças estatisticamente significativas entre o ouvido direito e o ouvido esquerdo apenas para a frequência de $250 \mathrm{~Hz}(p=0,043)$, com limiares mais baixos no ouvido esquerdo comparativamente com o ouvido direito (conforme a Fig. 1).

Os valores médios da perda tonal média (PTM), para ouvidos direito e esquerdo são respetivamente 35,6 dB (DP = 10,9; min. = 12,5; máx. = 50) e 34,9 dB $(D P=9,2$; min. = 11,25; máx. $=45)$, não havendo diferenças estatisticamente significativas entre os dois ouvidos ( $p=0,295)$. Verificou-se ainda que, relativamente ao ouvido esquerdo, $65 \%$ dos idosos apresentam uma perda de audição ligeira e 20\% uma perda auditiva de grau moderado. Relativamente ao ouvido direito, 45\% apresentam uma perda de audição ligeira e 45\% uma perda auditiva de grau moderado.

No teste de fala no ruído, para o ouvido esquerdo, a média total de acertos foi de 23,9\% (DP = 19,8\%; min. = 0\%; máx. $=73 \%$ ) (Fig. 2). Os resultados obtidos evidenciaram ainda uma média de acertos de 21,5\% (DP = 19,8\%; min. $=0 \%$; máx. $=70 \%$ ) para uma relação sinal/ruído de $10 \mathrm{~dB}$ tendo um aumento significativo $(p<0,001) \mathrm{com}$ a relação sinal/ruído de $15 \mathrm{~dB}$ tendo-se obtido uma média de acertos 26,3\% (DP = 2,4\%; min. = 0\%; máx. = 77\%).

Relativamente ao ouvido direito, obteve-se uma média de 17,3\% de acertos (DP = 14,9\%; min. = 0\%; máx. = 50\%) para uma relação sinal/ruído de $10 \mathrm{~dB}$ e de $27,85 \%$ de acertos ( $D P=19,1 \%$; min. $=0 \%$; máx. $=70 \%)$ tendo um aumento significativo ( $p=0,002)$ com a relação sinal/ ruído de $15 \mathrm{~dB}$. Verificou-se ainda um total de acertos de 22,5\% (DP = 16,5\%; min. = 0\%; máx. = 58\%).

Não se verificaram diferenças estatisticamente significativas entre os dois ouvidos ( $p=0,088 ; p=0$ 0,321 e $p=0,432$ para $10 \mathrm{~dB}, 15 \mathrm{~dB}$ e total respetivamente).

No que respeita à relação entre o teste de fala no ruído e a perda tonal média (PTM) verificam-se correlações moderadas negativas, no ouvido direito a $10 \mathrm{~dB}$ e $15 \mathrm{~dB}$, e fortes no ouvido esquerdo e no ouvido direito no total, ou seja, nesta amostra com o aumento da PTM diminui a percentagem de acertos com valores estatisticamente significativos ( $p<0,001)$, tal como mostra a Tabela 1. 


\section{DISCUSSÃO}

Tendo em conta os resultados obtidos no audiograma tonal simples e a média dos limiares auditivos da amostra estudada verificamos que a maior parte dos indivíduos apresenta uma perda de audição de grau ligeiro: 45\% no ouvido direito e $65 \%$ no ouvido esquerdo, segundo a norma 02 do Bureau International d'Audiophonologie-BIAP (1997),22 com uma média da perda tonal média de 35,6 dB no ouvido direito e 34,9 dB no ouvido esquerdo. Este perfil da curva audiométrica é compatível com a presbiacúsia, havendo um agravamento dos limiares auditivos estatisticamente significativo ( $p<0,001 \mathrm{em}$ ambos os ouvidos) com o aumento das frequências testadas, a partir da frequência $500 \mathrm{~Hz}$. A curva audiométrica mostra, qualitativamente, uma melhoria dos limiares auditivos dos $250 \mathrm{~Hz}$ para os $500 \mathrm{~Hz}$, em ambos os ouvidos, com declínio dos limiares auditivos nas frequências acima dos $500 \mathrm{~Hz}$; ou seja, nesta amostra verifica-se um aumento do limiar auditivo associado ao aumento da frequência.

Apesar do ouvido esquerdo apresentar melhores limiares do que o ouvido direito, apenas se observou significância estatística $(p=0,043)$, nos $250 \mathrm{~Hz}$, sendo o perfil da curva audiométrica muito semelhante em ambos os ouvidos (Fig. 1). Este perfil mostra já o efeito do enveIhecimento no sistema auditivo, com maior privação das frequências agudas.

Perante uma perda de audição de grau ligeiro ou mesmo moderado, com este tipo de curva audiométrica, onde as frequências agudas estão mais afetadas, era suposto os sujeitos apresentarem dificuldades na discriminação dos fonemas correspondentes a esta zona frequencial. Porém não seria de esperar que os sujeitos obtivessem uma percentagem de acertos tão baixa no teste de fala no ruído para uma relação sinal/ruído de $10 \mathrm{~dB}$ e de $15 \mathrm{~dB}$ sendo que, a média dos acertos obtida para ambos os ouvidos e, independentemente da relação sinal/ ruído, foi sempre inferior aos 50\% (Fig. 2). Este resultado demonstra que também o processamento auditivo central está afetado. Esta perturbação pode estar diretamente relacionada com o processo de envelhecimento, mas também com a perda auditiva mesmo na presença de uma perda auditiva de grau ligeiro.

Os resultados obtidos no estudo de Caporali e Silva, ${ }^{23}$ corroboram com os resultados obtidos neste estudo uma vez que os indivíduos com perda auditiva, independentemente do seu grau, apresentam uma dificuldade bastante significativa na interpretação e compreensão da fala em situações de ruído. ${ }^{23}$
Apesar das alterações do sistema auditivo periférico serem apontadas como a principal causa das dificuldades auditivas e de comunicação nas pessoas idosas, também a deterioração ao nível do processamento auditivo central conduz ao comprometimento da capacidade de manter um discurso coerente. ${ }^{24}$ Deste modo, as alterações a nível do sistema auditivo central em conjunto com as alterações a nível periférico, resultarão na dificuldade acrescida na compreensão do discurso da pessoa idosa, o que irá prejudicar as suas rotinas diárias, como por exemplo a ida ao supermercado ou ao café, que consequentemente levará ao isolamento da pessoa mais velha. ${ }^{23,24}$

A Academia Americana de Audiologia publicou uma revisão da literatura sobre presbiacúsia central, concluindo que as evidências sustentam a existência de uma presbiacúsia central que surge de uma combinação de fatores envolvendo questões relacionadas à idade e à doença, incluindo a função cognitiva ${ }^{25}$ sendo esta uma área que necessita de mais investigação e estudo no nosso país.

Apesar da amostra inicial ser constituída por apenas 20 idosos, não significa que o número de indivíduos com alterações ao nível da audição neste centro de dia da zona centro do país seja reduzido, pelo contrário. Há um número significativo de idosos com surdez sensorioneural bilateral de grau severo e profundo, motivo pelo qual foram excluídos do estudo dada a impossibilidade de aplicação do teste de fala no ruído, como referido anteriormente.

Perante os resultados obtidos no nosso estudo seria de esperar que estes indivíduos com défice auditivo e, com as dificuldades que todos apresentaram na discriminação da fala no ruído, estivessem a seguir um programa de reabilitação auditiva individual, com recurso à adaptação protésica, devidamente ajustado ao estilo de vida de cada sujeito o qual deveria incluir sessões de treino auditivo e o seu respetivo acompanhamento. ${ }^{14-16}$ No entanto, nenhum deles estava a cumprir programa de reabilitação auditiva nem tão pouco sensibilizado para a sua importância devido ao baixo status socioeconómico dos participantes bem como devido ao custo associado à aquisição dos aparelhos auditivos.

Kraus e Anderson ${ }^{26}$ esclarecem que quando avaliamos e aconselhamos pessoas idosas com dificuldades de audição, devemos ponderar sempre a existência de problemas centrais bem como a influência de toda a via auditiva no processamento da informação e não apenas a parte periférica. ${ }^{26}$

A avaliação do processamento auditivo em Portugal é recente e está mais direcionada para a população mais 
jovem. Porém não podemos descorar as questões centrais nesta população, que não serão diagnosticadas precocemente uma vez que não é prática clínica regular a avaliação do processamento auditivo central nesta faixa etária. Torna-se, por conseguinte imperativo a mudança de paradigma e a consciencialização dos profissionais de saúde envolvidos neste processo nomeadamente, médicos otorrinolaringologistas, audiologistas e terapeutas da fala para a importância da avaliação do processamento auditivo no idoso, por forma a encaminhar estes sujeitos para programas de reabilitação e/ou treino auditivo.

Vários estudos provam que o treino auditivo nos idosos apresenta melhorias significativas no reconhecimento dos sons, das palavras e de frases, mesmo em situações de ruido competitivo. ${ }^{23}$ É possível com apenas algumas sessões de treino auditivo melhorar a discriminação da fala no ruído mesmo em sujeitos com perda auditiva. ${ }^{20,26,27}$

Tendo em conta todos estes fatores mencionados, torna-se urgente a implementação de protocolos cuja avaliação audiológica contemple a avaliação periférica e central, na população mais velha permitindo o seu encaminhamento para programas de reabilitação auditiva ajustados às necessidades individuais de cada sujeito que podem incluir sessões de treino auditivo. Todo este processo irá garantir o sucesso dos programas de reabilitação auditiva, permitindo diminuir o número de pessoas mais velhas que vivem no isolamento e os sintomas depressivos característicos desta população.

Estes são resultados preliminares pelo que seria pertinente a realização de mais estudos sobre processamento auditivo central, na população idosa portuguesa.

Os principais limites deste estudo foi o reduzido número da amostra, o que impossibilita a generalização destes resultados para a população mais velha portuguesa e a ausência de estudos similares em Portugal. Seria interessante a realização de mais estudos que avaliem o processamento auditivo central em pessoas idosas, designadamente que utilizem o teste de fala no ruído modo a sustentar, ou não, os resultados do presente estudo.

\section{CONCLUSÃO}

Com este estudo, podemos concluir que a população mais velha, muitas das vezes depara-se com dificuldades na compreensão da fala e do discurso, principalmente em situações de ruído. Por conseguinte, a perda de au- dição e a dificuldade na compreensão da fala no ruído caracterizam o estado de degradação da saúde auditiva da população idosa em Portugal e a falta de investimento em programas de reabilitação auditiva. Assim, torna-se urgente a implementação de protocolos cuja avaliação audiológica contemple a avaliação periférica e central, na população mais velha permitindo o seu encaminhamento para programas de reabilitação auditiva ajustados às necessidades individuais de cada sujeito que podem incluir sessões de treino auditivo. Todo este processo irá garantir o sucesso dos programas de reabilitação auditiva, permitindo diminuir o número de pessoas mais velhas que vivem no isolamento e os sintomas depressivos característicos desta população.

CONFLITOS DE INTERESSE: Os autores declaram não ter qualquer conflito de interesse na realização do presente trabalho.

FONTES DE FINANCIAMENTO: Este trabalho é cofinanciado pelo Fundo Europeu de Desenvolvimento Regional (FEDER), através do Acordo de Parceria Portugal 2020 - Programa Operacional Regional do Centro (CENTRO 2020), no âmbito do projeto CENTRO-01-0145-FEDER-023369AGA@4life: AGA - Abordagem Geriátrica Ampla na promoção de um envelhecimento ativo e saudável - implementação de um programa de intervenção integrado e multidisciplinar.

CONFIDENCIALIDADE DOS DADOS: Os autores declaram ter seguido os protocolos da sua instituição acerca da publicação dos dados de doentes.

PROTEÇÃO DE PESSOAS E ANIMAIS: Os autores declaram que os procedimentos seguidos na elaboração do presente trabalho estão em conformidade com as normas das comissões de investigação clínica e de ética, bem como da declaração de Helsínquia e da Associação Médica Mundial.

PROVENIÊNCIA E REVISÃO POR PARES: Não comissionado; revisão externa por pares.

CONFLICTS OF INTEREST: The authors declare that they have no conflicts of interest.

FINANCING SUPPORT: This work is co-financed by the Fundo Europeu de Desenvolvimento Regional (FEDER), through the Portugal Partnership Agreement 2020 - Programa Operacional Regional do Centro (CENTRO 2020), within the project CENTRO-01-0145-FEDER-023369AGA@4life: AGA - Abordagem Geriátrica Ampla for the promotion of a healthy and active ageing - implementation of a multidisciplinary and integrated intervention programme. 
CONFIDENTIALITY OF DATA: The authors declare that they have followed the protocols of their work center on the publication of data from patients.

PROTECTION OF HUMAN AND ANIMAL SUBJECTS: The authors declare that the procedures followed were in accordance with the regulations of the relevant clinical research ethics committee and with those of the Code of Ethics of the World Medical Association (Declaration of Helsinki).

PROVENANCE AND PEER REVIEW: Not commissioned; externally peer reviewed.

\section{REFERÊNCIAS}

1. Martin JS, Jerger JF. Some effects of aging on central auditory processing. J Rehabil Res Dev. 2014; 42:25-44. doi: 10.1682/ JRRD.2004.12.0164.

2. Ruschel CV, Carvalho CR, Guarinello AC. The efficiency of an auditory rehabilitation program in elderly people with presbicusys and their family. J Soc Bras Fonoaudiologa. 2007; 12:958. doi: 10.1590/S1516-80342007000200005.

3. Veras R, Mattos L. Audiologia do envelhecimento: revisão da literatura e perspetivas atuais. Rev Bras Otorrinolaringol. 2007; 73: 128-34. doi: 10.1590/S0034-72992007000100021.

4. Buss LH, Graciolli LS, Rossi AG. Auditory processing in elderly: implications and solutions. Rev CEFAC. 2010; 12: 146-51. doi: 10.1590/S1516-18462009005000066.

5. Peixoto MC, Miranda C, Bento M, Oliveira S, Silva VC. Protocolo de Avaliação da Discriminação da Fala no Ruído com Implantes Auditivos. Gazeta Méd. 2018; 3: 196-200. doi: 10.29315/gm.v5i3.186.

6. American Speech-Language-Hearing Association. Central Auditory Processing: Current Status of Research and Implications for Clinical Practice. Am J Audiol. 1995; 5: 41-54.

7. American Speech Language Hearing Association. (Central) Auditory Processing Disorders. [consultado jan 2019] Disponível em: URL: http://www.asha.org/members/deskref-journals/ deskref/default.

8. Nunes CL, Desgualdo L, Carvalho GS. Construção e validação dos testes fala com ruído (FR) e dicótico de dígitos (DD) para aplicação em crianças portuguesas. Rev Port Otorrinolaringol Cir Cérvico-Fac. 2011; 49: 23-7.

9. Neves VT, Feitosa MA. Controversies or complexity in the relationship between temporal auditory processing and aging? Rev Bras Otorrinolaringol. 2003; 69: 242-9. doi: 10.1590/ S0034-72992003000200015.

10. Bellis T. Assessment and Management of Central Auditory Processing Disorders in the Educational Setting: From Science to Practice. San Diego: Singular Publishing; 1996.

11. Katz J, Wilde L. Desordens do processamento auditivo. In: Katz J. Tratado de audiologia clínica. São Paulo: Manole; 1996.p. 486-98.

12. Gonçales AS, Curry ML. Assessment of two central auditory test in elderly patients without hearing complaints. Braz J Otorhinolaryngol. 2011; 77: 24-32.

13. Moore D, Rosen S, Bamiou D, Campbell N, Sirimanna T. Evolving concepts of developmental auditory processing disorder (APD): A British Society of Audiology APD Special Interest Group. Int J Audiol. 2013; 52: 3-13. doi: 0.3109 / 14992027.2012 .723143$.
14. Rivabem K. Linguagem Escrita e Distúrbios do Processamento Auditivo Central: Uma Relação de Casualidade Contraditória [dissertação] Curitiba: Universidade Tuiuti do Panamá; 2006.

15. Vellozo FF, Filha VA, Costa MJ, Biaggio EP, Garcia MV. Teste de identificação de sentenças sintéticas com mensagem competitiva ipsilateral pediátrico: revisão narrativa sobre a sua aplicabilidade. Rev CEFAC. 2015; 17: 1604-16. doi: 10.1590/1982021620151752315.

16. Brasil PD, Schochat E. Eficácia do treinamento auditivo utilizando o software Programa de Escuta no Ruído (PER) em escolares com transtorno do processamento auditivo e baixo desempenho escolar. Rev CoDAS. 2018; 30: 1-6. doi: 10.1590/2317-1782/20182017227.

17. Musiek F. Auditory training and CAPD: a short history. Hear J. 2006; 59: 52. doi: 10.1055/s-0035-1564458.

18. Musiek F, Chermak G. Handbook of (Central) Auditory Processing Disorder. San Diego: Plural Publishing Inc; 2007.

19. Martins JH, Teixeira A. Bateria de Avaliação de Processamento auditivo para o português Europeu (BAPA-PE). Lisboa: Círculo Médico/Widex; 2018.

20. Silva CM, Fernandes C, Alves AR, Prata C, Rocha C, Martins J. A influência do treino auditivo na comunicação do idoso. In: Abordagem geriátrica ampla na promoção de um envelhecimento ativo e saudável: Componentes do modelo de intervenção aga@4Life. Coimbra: IPC-Inovar Para Crescer; 2019. p. 75-86.

21. Pereira LD, Schochat E. Testes Auditivos Comportamentais para Avaliação do Processamento Auditivo Central. São Paulo: Pró-Fono; 2011

22. BIAP: Audiometrische classificatie van Gehoorstoornissen. Lisboa: International Bureau for Audiophonologie; 1997 [consultado março 2019]. Available from: http://www.biap.org/es/.

23. Caporali SA, Silva JA. Reconhecimento da fala no ruído em jovens e idosos com perda auditiva. Rev Bras Otorrinolaringol. 2004; 70: 525-32. doi: 10.7162/S180948722012000100002

24. Dubno JR. Benefits of Auditory Training for Aided Listening by Older Adults. Am J Audiol. 2013; 22: 335-38. doi: 10.1044/1059-0889(2013/12-0080).

25. Humes LE, Dubno JR, Gordon-Salant S, Lister JJ, Cacace AT, Cruickshanks KJ, et al. Central presbycusis: a review and evaluation of the evidence. J Am Acad Audiol. 2012; 23: 63566. doi: 10.3766/jaaa.23.8.5.

26. Kraus N, Anderson S. The Effects of Aging on Auditory Processing Hear J; 66:36. doi: 10.1097/01HJ000042577480002.

27. Morais AA, Rocha-Muniz CN, Schochat E. Efficacy of auditory training in elderly subjects. Front Aging Neurosci. 2015; 7: 78. doi: 10.3389/fnagi.2015.00078. 